\title{
Identifying on admission patients likely to develop acute kidney injury in hospital
}

\author{
Anastasios Argyropoulos ${ }^{*}$, Stuart Townley ${ }^{2}$, Paul M. Upton ${ }^{3}$, Stephen Dickinson ${ }^{3}$ and Adam S. Pollard ${ }^{3}$
}

\begin{abstract}
Background: The incidence of Acute Kidney Injury (AKI) continues to increase in the UK, with associated mortality rates remaining significant. Approximately one fifth of hospital admissions are associated with AKI and approximately a third of patients with AKI in hospital develop AKI during their time in hospital. A fifth of these cases are considered avoidable. Early risk detection remains key to decreasing AKI in hospitals, where sub-optimal care was noted for half of patients who developed AKI.

Methods: Electronic anonymised data for adults admitted into the Royal Cornwall Hospitals Trust (RCHT) between 18th March and 31st December 2015 was trimmed to that collected within the first $24 \mathrm{~h}$ of hospitalisation. These datasets were split according to three separate time periods: data used for training the Takagi-Sugeno Fuzzy Logic Systems (FLS) and the multivariable logistic regression (MLR) models; data used for testing; and data from a later patient spell used for validation.

Three fuzzy logic models and three MLR models were developed to link characteristics of patients diagnosed with a maximum stage AKI within 7 days of admission: the first models to identify any AKI Stage (FLS I, MLR I), the second for patterns of AKI Stage 2 or 3 (FLS II, MLR II), and the third to identify AKI Stage 3 (FLS III, MLR III). Model accuracy is expressed by area under the curve (AUC).

Results: Accuracy for each model during internal validation was: FLS I and MLR I (AUC 0.70, 95\% Cl: 0.64-0.77); FLS II (AUC 0.77, 95\% Cl: 0.69-0.85) and MLR II (AUC 0.74, 95\% Cl: 0.65-0.83); FLS III and MLR III (AUC 0.95, 95\% Cl: 0.92-0.98).

Conclusions: FLS II and FLS III (and the respective MLR models) can identify with a high level of accuracy patients at high risk of developing AKI in hospital. These two models cannot be properly assessed against prior studies as this is the first attempt at quantifying the risk of developing specific Stages of AKI for a broad cohort of both medical and surgical inpatients. FLS I and MLR I performance is comparable to other existing models.
\end{abstract}

Keywords: Acute kidney injury, AKI, Fuzzy logic, Multivariable logistic regression, Risk factors, Forward selection

\section{Background}

Acute Kidney Injury (AKI) is emerging as an important clinical syndrome which is associated with poor clinical outcomes. AKI, previously known as Acute Renal Failure, refers to a sudden reduction in kidney function.

The Kidney Disease: Improving Global Outcomes (KDIGO) Acute Kidney Injury Work Group definitions of AKI have been adopted in the UK. This classification

\footnotetext{
* Correspondence: a.argyropoulos@soton.ac.uk

I, Dr Anastasios Argyropoulos, as corresponding author can confirm that I had full access to all the data in this study and had final responsibility for the decision to submit for publication

${ }^{1}$ Centre for Implementation Science, Faculty of Health Sciences, University of Southampton, Southampton SO17 1BJ, UK

Full list of author information is available at the end of the article
}

identifies three stages of severity of AKI, based on rises in serum creatinine or reduction in urine output (Table 1) $[1,2]$. At the Royal Cornwall Hospitals Trust (RCHT) clinicians predominantly use creatinine change more often than urine output to classify AKI Stage, and in this study, we only used creatinine change.

The majority of AKI is caused by sepsis and hypovolaemia. Other causes include renal tract obstruction and intrinsic renal disease. Around 1 in 5 adult hospital admissions are associated with AKI [3]. AKI is present on admission in around $60 \%$ of all patients with AKI detected in hospital [4]. AKI is associated with high mortality rates; from 8 to $18 \%, 22-33 \%$, and $32-36 \%$ mortality for patients with AKI Stages 1, 2, and 3

(c) The Author(s). 2019 Open Access This article is distributed under the terms of the Creative Commons Attribution 4.0 International License (http://creativecommons.org/licenses/by/4.0/), which permits unrestricted use, distribution, and 
Table 1 Measurements used to classify the three Stages of AKI

\begin{tabular}{lll}
\hline Stage & Serum Creatinine & Urine output \\
\hline 1 & $1.5-1.9 \times$ baseline creatinine, or & $<0.5 \mathrm{ml} / \mathrm{kg} / \mathrm{hr}$. for $6-12 \mathrm{~h}$ \\
& $\geq 26.4 \mu \mathrm{mol} / \mathrm{l}$ within $48 \mathrm{~h}$ & \\
2 & $2.0-2.9 \times$ baseline creatinine & $<0.5 \mathrm{ml} / \mathrm{kg} / \mathrm{hr}$. for $\geq 12 \mathrm{~h}$ \\
3 & $\geq 3.0$ times the baseline, or $\geq$ & $<0.3 \mathrm{ml} / \mathrm{kg} / \mathrm{hr}$. for $\geq 24 \mathrm{~h}$, \\
& $\begin{array}{l}354 \mu \mathrm{mol} / \mathrm{l}, \text { or initiation of renal } \\
\text { replacement therapy }\end{array}$ & or anuria $\geq 12 \mathrm{~h}$ \\
&
\end{tabular}

respectively, whilst in the absence of AKI, mortality runs at 2\% [4-6]. Consequently, in England AKI may be associated with as many as 40,000 excess deaths each year [6]. Hospital inpatient AKI care has been estimated to cost $£ 1$ billion each year, and effective prevention of AKI could save up to $£ 200$ million per annum [6].

The 2009 National Confidential Enquiry into Patient Outcome and Death (NCEPOD) AKI report found that care could have been improved for $50 \%$ of patients [7]. Since the NCEPOD report, great strides have been made in hospital AKI care, including the introduction in UK hospitals of an AKI algorithm to detect AKI and national advice on what an AKI Bundle should include [2, 8]. Clinically recognised risk factors for AKI include modifiable factors (e.g. use of iodinated contrast, use of certain medications) and non-modifiable factors (e.g. age, the presence of chronic kidney disease) [9]; combinations of these modifiable and non-modifiable factors appear to multiply risks of developing AKI. Roberts et al. describe that age alone is closely associated with the number of AKI risk factors patients have, as well as itself being associated with AKI [10].

Given that approximately a third of patients with AKI in hospital develop AKI during their stay in hospital, two-thirds of patients with AKI in hospital have AKI at the time of admission [4]. So, there is an opportunity to prevent the development of AKI in a large number of patients: in cases of AKI developed whilst in hospital, $20 \%$ are avoidable [7]. An AKI risk prediction tool could identify patients at high risk of AKI and provide the attending medical teams the opportunity to prevent the development of AKI by intervening in patients' current care. For example, staff would be alerted to the risk faced by individual patients, prompting a review of the patient's current clinical condition, and current medication, which may lead to increased clinical monitoring or a change in treatment. This could cut rates of AKI, leading to a reduction in morbidity, mortality, and length of stay.

We describe an attempt at predicting which patients will go on to develop AKI in hospital, and specifically which Stage of AKI. This is undertaken using methods based around fuzzy logic which are then calibrated against more conventional approaches.

\section{Methods}

Three fuzzy logic systems (FLS) (FLS I, II, III) and three multivariable logistic regression (MLR) models (MLR I, II, III) were constructed to stratify patient risk for developing AKI (any Stage), either Stage 2 or 3 AKI, or Stage 3 AKI respectively during a stay in hospital. Each model was parameterised with data from a time period and validated using data from a later period. Data was provided by RCHT. Each FLS was distinguishable from the other due to the different sets of risk factors used in defining FLS I, II and III respectively.

\section{Data collection}

Research presented here draws on data relating to patients admitted into RCHT in the UK (www.royalcornwall.nhs.uk). RCHT serves roughly 430,000 people and employs about 5000 staff with a budget of approximately $£ 380$ million. Across its three sites it admits approximately 59,000 emergencies (including maternity and births) and 9000 elective inpatients per year into 750 beds.

Construction of a FLS involves three stages: training, testing, and validation. Each FLS was trained using anonymised data for 5504 patients treated by RCHT between $18 / 03 / 2015$ and $30 / 09 / 2015$; tested using equivalent data for 937 patients from October 2015; and validated with RCHT data for 1020 patients treated during November and December 2015. The same process was followed for the development and validation of the MLR models.

\section{Risk factor definition and initial identification}

AKI was identified using serum creatinine levels only through the usual laboratory method at the hospital, i.e. the NHS England AKI algorithm identified the stage of AKI on all blood samples received in the laboratory [2]. The NHS England AKI algorithm is based on the KDIGO AKI classification (Table 1).

Risk factors for use in developing the models were selected following clinical discussion and literature review. The list of risk factors was reduced by variables not defined by data availability electronically for all inpatients. Consequently, 25 variables were initially selected for entry into each model.

For risk factors: platelets, white blood count (WBC), red blood count (RBC), haematocrit $(\mathrm{HCT})$, haemoglobin $(\mathrm{Hb})$, mean platelet volume (MPV), mean corpuscular volume $(\mathrm{MCV})$, sodium $(\mathrm{Na})$, potassium $(\mathrm{K})$, chloride (CL-), urea, creatinine and albumin, data records available between $24 \mathrm{~h}$ before admission and $24 \mathrm{~h}$ after admission for patients undergoing tests in that time window were kept. If the data records were available for both the day before admission and the day of admission, the record from the day before admission was selected. 
Given the absence of the exact processing time of test results, where multiple of the same type were undertaken per patient per day, a result was selected at random from the test of the same type performed. The remaining risk factors were available on admission, which were, for each patient:

- Age (years), gender (male or female), type of admission (medical or surgical)

- Prescription of non-steroidal anti-inflammatory drugs (NSAID), prescription of angiotensin converting enzyme inhibitors (ACEI)

- Comorbidities: diabetes, heart failure, chronic kidney disease (CKD) and vascular disease (VD) of coronary artery and heart, not including acute myocardial infarction and associated complications. Patients with these comorbidities were identified as those presenting with known pre-existing conditions.

Data were excluded from this study for patients under 18 years of age, for patients staying less than $48 \mathrm{~h}$ in hospital and for patients who developed AKI $>7$ days after admission. Incomplete records were also removed.

\section{Statistical analysis}

The association between dependent and independent variables aiming to identify the most suitable risk factors to be included in each model was examined by performing univariable and multivariable analyses using SPSS version 24.0. Initially, univariable logistic regression analysis was conducted to determine the odds ratios of AKI incidence for each risk factor. Next, multiple logistic regression models based on forward stepwise selection were developed by including risk factors with univariable $p$-values $<0.25$. A value of $p \leq 0.05$ was considered to be statistically significant.

\section{Fuzzy logic system development}

Takagi-Sugeno-Kang (TSK) or Sugeno fuzzy logic (FL) models based on subtractive clustering were developed using the Fuzzy Logic Toolbox within the framework of Matlab R2016a. The TSK FLS methodology was originally developed as an approach to represent a series of systems and functions by utilising input-output data [11, 12]. The absence of a systematic way to derive appropriate parameters for the FLS can be overcome with data clustering. Further details regarding the design of TSK FLS can be found in [13].The fuzzy modelling algorithm is portrayed in Additional file 1: Figure S1.

\section{Model performance measures}

The Area Under the Curve (AUC) was calculated for each Receiver Operating Characteristic (ROC) Curve to evaluate the effectiveness of the models [14]. Model performance was also evaluated using sensitivity (SN) and specificity (SP) with a single decision threshold determined for each model during testing and applied during the validation process based on the method that minimises the absolute value of Specificity-Sensitivity [15]. Analysis was performed using R (3.4.3) with packages "OptimalCutpoints" and "pROC" utilised in the calculation of the optimal values and performances [16, 17].

To assess whether any loss in model accuracy might be caused by the use of TSK FL, equivalent models for FLS I, II and III were assembled using Logistic Regression [15]. Logistic Regression has been widely applied in developing risk assessment frameworks in healthcare, and as such, is adept at "ground truthing" performance of each of the FLS models [18].

\section{Results}

\section{Patient characteristics}

A summary of the characteristics of patients with and without AKI is shown in Additional file 1: Table S1. Of 5504 patient admissions into RCHT, 216 (3.9\%) were diagnosed with any Stage AKI during hospitalisation and were included in the development of FLS I (training). For FLS I testing, the equivalent patient numbers were 937 and 89 (9.5\%); and for FLS I validation, 1020 and 65 (6.4\%) respectively. In general, AKI patients were older than those without AKI (mean (SD) age was 75.1 (14.2) years vs 66.7 (19.4) years), had a higher incidence of heart failure (30.3\% vs $13.5 \%)$ and CKD (34.6\% vs $13.5 \%)$ and a lower incidence of NSAID (2.7\% vs $7.0 \%)$ when compared to low risk patients.

Additional file 1: Table S3 has the equivalent patient characteristics for data feeding FLS II, with 94 (1.7\%) of 5504 patients contracting Stage 2 AKI or Stage 3 AKI used in training FLS II. For FLS II testing and validation, respectively $30(3.2 \%)$ of 937 patients and $28(2.7 \%)$ of 1020 patients developed Stage 2 AKI or Stage 3 AKI. Stage 2 AKI and Stage 3 AKI patients were older (age was $74.7(14.6)$ years vs $67.0(19.4))$ and were more likely to have heart failure $(28.3 \%$ vs $14 \%)$, diabetes $(27.6 \%$ vs $19 \%)$ and CKD (38.8\% vs $14.1 \%)$ when compared to low risk patients or patients who developed Stage 1 AKI.

Patient characteristics of patients at low AKI risk, patients diagnosed with Stage 1 AKI or Stage 2 AKI, along with patients who developed Stage 3 AKI are depicted in Additional file 1: Table S4. Thirty- six records (0.65\%) of patients who contracted Stage 3 AKI were used in the design of FLS III (training); 16 (1.7\%) diagnosed with Stage 3 AKI were used in FLS III calibration process (testing) and $14(1.4 \%)$ in the validation. Stage 3 AKI patients were older (age was 72.8 (15.4) years vs 67.1 (19.3)) and had a higher heart failure (25.8\% vs $14.2 \%)$ and CKD (45.5\% vs $14.3 \%$ ) prevalence when compared 
to low risk patients or patients who developed Stage 1 AKI or Stage 2 AKI.

Biochemical marker distributions and clinical characteristics are summarised in Additional file 1: Table S1, Table S2, Table S3 and Table S4.

\section{Risk factor selection}

\section{FLS I and MLR I}

Univariable analysis aimed at identifying risk factors to for inclusion in FLS I (Table 2) revealed that platelets $(p=0.041)$, RBC $(p<0.001)$, HTC $(p=0.001)$, Haemoglobin $(\mathrm{p}<0.001), \operatorname{MPV}(\mathrm{p}<0.001), \operatorname{MCV}(p=0.095)$, Sodium $(p=0.034)$, Potassium $(\mathrm{p}<0.001)$, Chloride $(p=$ $0.069)$, Urea $(\mathrm{p}<0.001)$, Creatinine $(\mathrm{p}<0.001)$, Albumin $(\mathrm{p}<0.001)$, age $(\mathrm{p}<0.001)$, gender $(p=0.027)$, NSAID $(p=0.009)$, ACEI $(p=0.037)$, diabetes $(\mathrm{p}=0.009)$, heart failure $(\mathrm{p}<0.001)$, CKD $(\mathrm{p}<0.001)$ and VD (of coronary artery and heart not including acute myocardial infarction and associated complications) ( $p=0.006)$ were associated with the development of any Stage AKI. Despite the fact that the $p$ value of the type of admission ( $p=$ $0.803>0.25)$ did not fulfil the selection criteria, it was considered by the authors that it could serve as a complementary predictor despite the univariable prefiltering [19]. Especially in the context of multiple regression, complementary predictors can be found that when used separately are unable to explain differences between classes (i.e. are not significant), but together they are [20]. This proved to be the case for the type of admission in the risk factor identification. Following forward selection, MPV $(p=0.033)$, Urea $(p=0.007)$, Albumin $(p<0.001)$, age $(\mathrm{p}<0.001)$, type of admission $(p=0.018)$, heart failure $(\mathrm{p}<0.001)$ and $\operatorname{CKD}(p=0.013)$ were retained for model development (Table 2).

\section{FLS II and MLR II}

Univariable analysis showed that RBC $(p=0.076)$, HCT $(p=0.066)$, Haemoglobin $(p=0.009)$, MPV $(p=0.008)$, Potassium $(p<0.001)$, Urea $(\mathrm{p}<0.001)$, Creatinine $(\mathrm{p}<$ $0.001)$, Albumin $(\mathrm{p}<0.001)$, age $(\mathrm{p}<0.001)$, NSAID $(p=$

Table 2 Risk factors associated with AKI (any stage) by univariable and multivariable analysis

\begin{tabular}{|c|c|c|c|c|c|c|}
\hline \multirow[t]{2}{*}{ Factors } & \multicolumn{3}{|c|}{ Univariable Analysis } & \multicolumn{3}{|c|}{ Multivariable Analysis } \\
\hline & $\overline{\mathrm{OR}}$ & $95 \% \mathrm{Cl}$ & $\overline{p \text {-Value }}$ & $\overline{\mathrm{OR}}$ & $95 \% \mathrm{Cl}$ & $p$-Value \\
\hline Platelets $\left(10^{\mathrm{a}} 9 / \mathrm{L}\right)$ & 0.999 & $0.997-1.000$ & 0.041 & & & \\
\hline WBC $\left(10^{\mathrm{a}} 9 / \mathrm{L}\right)$ & 1.006 & $0.988-1.025$ & 0.487 & & & \\
\hline $\operatorname{RBC}\left(10^{12} / \mathrm{L}\right)$ & 0.707 & $0.589-0.848$ & $<0.001$ & & & \\
\hline $\mathrm{HCT}(\mathrm{L} / \mathrm{L})$ & 0.030 & $0.004-0.236$ & 0.001 & & & \\
\hline $\mathrm{Hb}(\mathrm{g} / \mathrm{dL})$ & 0.988 & $0.982-0.993$ & $<0.001$ & & & \\
\hline MPV (fL) & 1.181 & $1.079-1.294$ & $<0.001$ & 1.109 & $1.008-1.221$ & 0.033 \\
\hline MCV (fL) & 1.017 & $0.997-1.036$ & 0.095 & & & \\
\hline $\mathrm{Na}(\mathrm{mmol} / \mathrm{L})$ & 0.973 & $0.949-0.998$ & 0.034 & & & \\
\hline $\mathrm{K}(\mathrm{mmol} / \mathrm{L})$ & 1.482 & $1.193-1.841$ & $<0.001$ & & & \\
\hline $\mathrm{CL}-(\mathrm{mmol} / \mathrm{L})$ & 0.980 & $0.958-1.002$ & 0.069 & & & \\
\hline Urea (mmol/L) & 1.066 & $1.048-1.084$ & $<0.001$ & 1.028 & $1.008-1.049$ & 0.007 \\
\hline Creatinine (mmol/L) & 1.002 & $1.001-1.003$ & $<0.001$ & & & \\
\hline Albumin (g/L) & 0.936 & $0.916-0.955$ & $<0.001$ & 0.953 & $0.931-0.976$ & $<0.001$ \\
\hline Age (years) & 1.035 & $1.025-1.045$ & $<0.001$ & 1.023 & $1.013-1.034$ & $<0.001$ \\
\hline Gender & 1.015 & $1.002-1.029$ & 0.027 & & & \\
\hline Chronic_OP (days) & 0.999 & $0.991-1.007$ & 0.716 & & & \\
\hline Chronic_IP (days) & 1.060 & $0.807-1.392$ & 0.674 & & & \\
\hline Admission type & 0.964 & $0.722-1.286$ & 0.803 & 1.440 & $1.064-1.949$ & 0.018 \\
\hline NSAID & 0.303 & $0.124-0.741$ & 0.009 & & & \\
\hline ACEI & 1.445 & $1.023-2.041$ & 0.037 & & & \\
\hline Diabetes & 1.514 & $1.108-2.068$ & 0.009 & & & \\
\hline Heart Failure & 2.920 & $2.168-3.932$ & $<0.001$ & 1.905 & $1.378-2.633$ & $<0.001$ \\
\hline CKD & 3.117 & $2.319-4.189$ & $<0.001$ & 1.560 & $1.099-2.213$ & 0.013 \\
\hline$V D^{a}$ & 1.523 & $1.131-2.052$ & 0.006 & & & \\
\hline
\end{tabular}

of coronary artery and heart, not including acute myocardial infarction and associated complications 
$0.152)$, ACEI $(p=0.201)$, diabetes $(p=0.017)$, heart failure $(p=0.001)$, CKD $(p<0.001)$ and VD (of coronary artery and heart not including acute myocardial infarction and associated complications) $(p=0.214)$ predicted patients diagnosed with Stage 2 or Stage 3 AKI (Table 3). As explained above, the type of admission $(p=0.673)$ was once again included in the multivariable analysis. However, following forward selection, Urea $(p<0.001)$, Albumin $(p=0.002)$ and age $(p=0.016)$ were identified as the most suitable risk factors (Table 3 ).

\section{FLS III and MLR III}

$\operatorname{MPV}(p=0.006)$, Potassium $(p<0.001)$, Chloride $(p=$ $0.15)$, Urea $(\mathrm{p}<0.001)$, Creatinine $(\mathrm{p}<0.001)$, Albumin $(p=0.011)$ and CKD $(\mathrm{p}=0.002)$ were identified as potential predictors of Stage 3 AKI using univariable analysis (Table 4). Similarly to the process followed in the risk factor selection for multivariable analysis for FLS I and FLS II, age $(p=0.261)$ and type of admission $(p=$ 0.340 ) were added to the variables identified by the univariable analysis. Following forward selection, Chloride $(p=0.001)$, Creatinine $(\mathrm{p}<0.001)$ and Albumin $(p=$ 0.023 ) were included in the model (Table 4).

\section{Model development and performance}

By applying the design process depicted in the Additional file 1: (Figure S1), for each model scope the FLS with the best performance during the testing process was identified using subtractive clustering. For FLS I optimal performance was reached when each input had two fuzzy sets based on the clustering performed and two rules governed the fuzzy system's function (fuzzy model). FLS II performed best when each input had three fuzzy sets based on the clustering performed and three rules governed the fuzzy system's function (fuzzy model). FLS III performance was maximised when each input had four fuzzy sets based on the clustering performed and four rules governed the fuzzy system's function (fuzzy model).

Table 3 Risk factors associated with AKI (Stage 2 or Stage 3) by univariable and multivariable analysis

\begin{tabular}{|c|c|c|c|c|c|c|}
\hline \multirow[t]{2}{*}{ Factors } & \multicolumn{3}{|c|}{ Univariable Analysis } & \multicolumn{3}{|c|}{ Multivariable Analysis } \\
\hline & $\overline{O R}$ & $95 \% \mathrm{Cl}$ & $p$-Value & $\overline{O R}$ & $95 \% \mathrm{Cl}$ & $p$-Value \\
\hline Platelets $\left(10^{\mathrm{a}} 9 / \mathrm{L}\right)$ & 0.999 & $0.997-1.001$ & 0.414 & & & \\
\hline WBC $\left(10^{\mathrm{a}} 9 / \mathrm{L}\right)$ & 1.005 & $0.978-1.033$ & 0.718 & & & \\
\hline $\operatorname{RBC}\left(10^{12} / \mathrm{L}\right)$ & 0.780 & $0.592-1.026$ & 0.076 & & & \\
\hline $\mathrm{HCT}(\mathrm{L} / \mathrm{L})$ & 0.054 & $0.002-1.212$ & 0.066 & & & \\
\hline $\mathrm{Hb}(\mathrm{g} / \mathrm{dL})$ & 0.988 & $0.980-0.997$ & 0.009 & & & \\
\hline MPV (fL) & 1.174 & $1.044-1.321$ & 0.008 & & & \\
\hline $\mathrm{MCV}(\mathrm{fL})$ & 1.002 & $0.973-1.032$ & 0.887 & & & \\
\hline $\mathrm{Na}(\mathrm{mmol} / \mathrm{L})$ & 0.996 & $0.957-1.036$ & 0.842 & & & \\
\hline $\mathrm{K}(\mathrm{mmol} / \mathrm{L})$ & 1.854 & $1.372-2.507$ & $<0.001$ & & & \\
\hline $\mathrm{CL}-(\mathrm{mmol} / \mathrm{L})$ & 0.994 & $0.961-1.029$ & 0.740 & & & \\
\hline Urea (mmol/L) & 1.071 & $1.048-1.094$ & $<0.001$ & 1.055 & $1.032-1.079$ & $<0.001$ \\
\hline Creatinine (mmol/L) & 1.003 & $1.002-1.004$ & $<0.001$ & & & \\
\hline Albumin (g/L) & 0.932 & $0.904-0.961$ & $<0.001$ & 0.948 & $0.917-0.981$ & 0.002 \\
\hline Age (years) & 1.027 & $1.014-1.041$ & $<0.001$ & 1.017 & $1.003-1.032$ & 0.016 \\
\hline Gender & 0.965 & $0.641-1.453$ & 0.865 & & & \\
\hline Chronic_OP (days) & 1.001 & $0.977-1.026$ & 0.940 & & & \\
\hline Chronic_IP (days) & 0.967 & $0.912-1.026$ & 0.268 & & & \\
\hline Admission type & 1.096 & $0.717-1.675$ & 0.673 & & & \\
\hline NSAID & 0.430 & $0.136-1.365$ & 0.152 & & & \\
\hline ACEI & 1.404 & $0.835-2.360$ & 0.201 & & & \\
\hline Diabetes & 1.730 & $1.101-2.719$ & 0.017 & & & \\
\hline Heart Failure & 2.210 & $1.390-3.515$ & 0.001 & & & \\
\hline CKD & 2.789 & $1.788-4.350$ & $<0.001$ & & & \\
\hline$V D^{a}$ & 1.336 & $0.846-2.108$ & 0.214 & & & \\
\hline
\end{tabular}

of coronary artery and heart, not including acute myocardial infarction and associated complications 
Table 4 Risk factors associated with Stage 3 AKI by univariable and multivariable analysis

\begin{tabular}{|c|c|c|c|c|c|c|}
\hline \multirow[t]{2}{*}{ Factors } & \multicolumn{3}{|c|}{ Univariable Analysis } & \multicolumn{3}{|c|}{ Multivariable Analysis } \\
\hline & $\mathrm{OR}$ & $95 \% \mathrm{Cl}$ & $p$-Value & OR & $95 \% \mathrm{Cl}$ & $p$-Value \\
\hline Platelets (10a/L) & 0.998 & $0.995-1.001$ & 0.264 & & & \\
\hline WBC (10ª/L) & 1.004 & $0.959-1.051$ & 0.874 & & & \\
\hline $\mathrm{RBC}\left(10^{12} / \mathrm{L}\right)$ & 1.041 & $0.659-1.645$ & 0.864 & & & \\
\hline $\mathrm{HCT}(\mathrm{L} / \mathrm{L})$ & 2.352 & $0.012-444.759$ & 0.749 & & & \\
\hline $\mathrm{Hb}(\mathrm{g} / \mathrm{dL})$ & 1.001 & $0.986-1.016$ & 0.941 & & & \\
\hline MPV (fL) & 1.222 & $1.060-1.408$ & 0.006 & & & \\
\hline $\mathrm{MCV}(\mathrm{fL})$ & 1.004 & $0.958-1.053$ & 0.856 & & & \\
\hline $\mathrm{Na}(\mathrm{mmol} / \mathrm{L})$ & 0.976 & $0.919-1.037$ & 0.429 & & & \\
\hline $\mathrm{K}(\mathrm{mmol} / \mathrm{L})$ & 2.246 & $1.449-3.481$ & $<0.001$ & & & \\
\hline $\mathrm{CL}-(\mathrm{mmol} / \mathrm{L})$ & 0.964 & $0.916-1.013$ & 0.150 & 0.968 & $0.949-0.987$ & 0.001 \\
\hline Urea (mmol/L) & 1.064 & $1.034-1.094$ & $<0.001$ & & & \\
\hline Creatinine (mmol/L) & 1.003 & $1.002-1.005$ & $<0.001$ & 1.003 & $1.002-1.004$ & $<0.001$ \\
\hline Albumin (g/L) & 0.937 & $0.892-0.985$ & 0.011 & 0.945 & 0.899-0.992 & 0.023 \\
\hline Age (years) & 1.011 & $0.992-1.030$ & 0.261 & & & \\
\hline Gender & 1.375 & $0.711-2.659$ & 0.344 & & & \\
\hline Chronic_OP (days) & 1.010 & $0.976-1.045$ & 0.573 & & & \\
\hline Chronic_IP (days) & 0.981 & $0.909-1.058$ & 0.612 & & & \\
\hline Admission type & 1.382 & $0.711-2.687$ & 0.340 & & & \\
\hline NSAID & 0.000 & $0.000-0.000$ & 0.994 & & & \\
\hline ACEl & 0.735 & $0.259-2.083$ & 0.562 & & & \\
\hline Diabetes & 1.215 & $0.552-2.674$ & 0.628 & & & \\
\hline Heart Failure & 0.967 & $0.375-2.493$ & 0.944 & & & \\
\hline CKD & 3.083 & $1.535-6.191$ & 0.002 & & & \\
\hline$V D^{a}$ & 1.159 & $0.544-2.472$ & 0.702 & & & \\
\hline
\end{tabular}

${ }^{a}$ of coronary artery and heart, not including acute myocardial infarction and associated complications

The FLSs' membership function parameters, rules and consequent parameters as well as the MLR models' coefficients, that were derived using the training dataset, are provided in Additional file 1: Table S5- Table S8.

\section{Model interpretation}

The ROC curves and their corresponding AUC values were calculated for each stage (training, testing, and validation) of each FLS (FLS I, II, and III) (Fig. 1) and of each MLR (MLR I, II, III) to demonstrate the ability of each model to discriminate between low risk and high risk AKI patients (Table 5). Comparative performance between each FLS and its respective MLR is shown in Table 5, Table 6 and Fig. 2.

For FLS I, AUC during validation was 0.7 (95\% CI: $0.64-0.77$ ) with SN and SP holding the values of 0.62 and 0.64 respectively. Positive predictive value (PPV) 0.1 and negative predictive value (NPV) was 0.96 (Table 5). Performance differed in MLR I only for SN and SP generated which were 0.57 and 0.69 respectively (Table 6).
In the case of FLS II, AUC (validation) was 0.77 (95\% CI: $0.69-0.85), \quad \mathrm{SN}=0.61, \quad \mathrm{SP}=0.73, \quad \mathrm{PPV}=0.06$ and $\mathrm{NPV}=0.99$. For the case of MLR II, the AUC dropped to 0.74 (95\% CI: $0.65-0.83$ ) versus FLS II whilst SP changed slightly to 0.72 (Table 5 , Table 6).

Model FLS III yielded an AUC during validation of 0.95 (95\% CI: $0.92-0.98$ ), producing a sensitivity of 1.0 and a specificity of 0.83 , with PPV $=0.08$ and $\mathrm{NPV}=1.0$. Although the AUC generated by MLR III mirrored that of FLS III, values of SP and PPV dropped to 0.79 and 0.06 respectively (Table 5 , Table 6 ).

\section{Discussion}

AKI is categorised into three Stages according to severity. The KDIGO stages of AKI are identified using creatinine measurements from blood tests, or from the reduction in urine output. Other methods to identify AKI such as using novel biomarkers are not used in practice, are evolving, and so were not analysed as part of this study [21]. Current practice only assesses the 

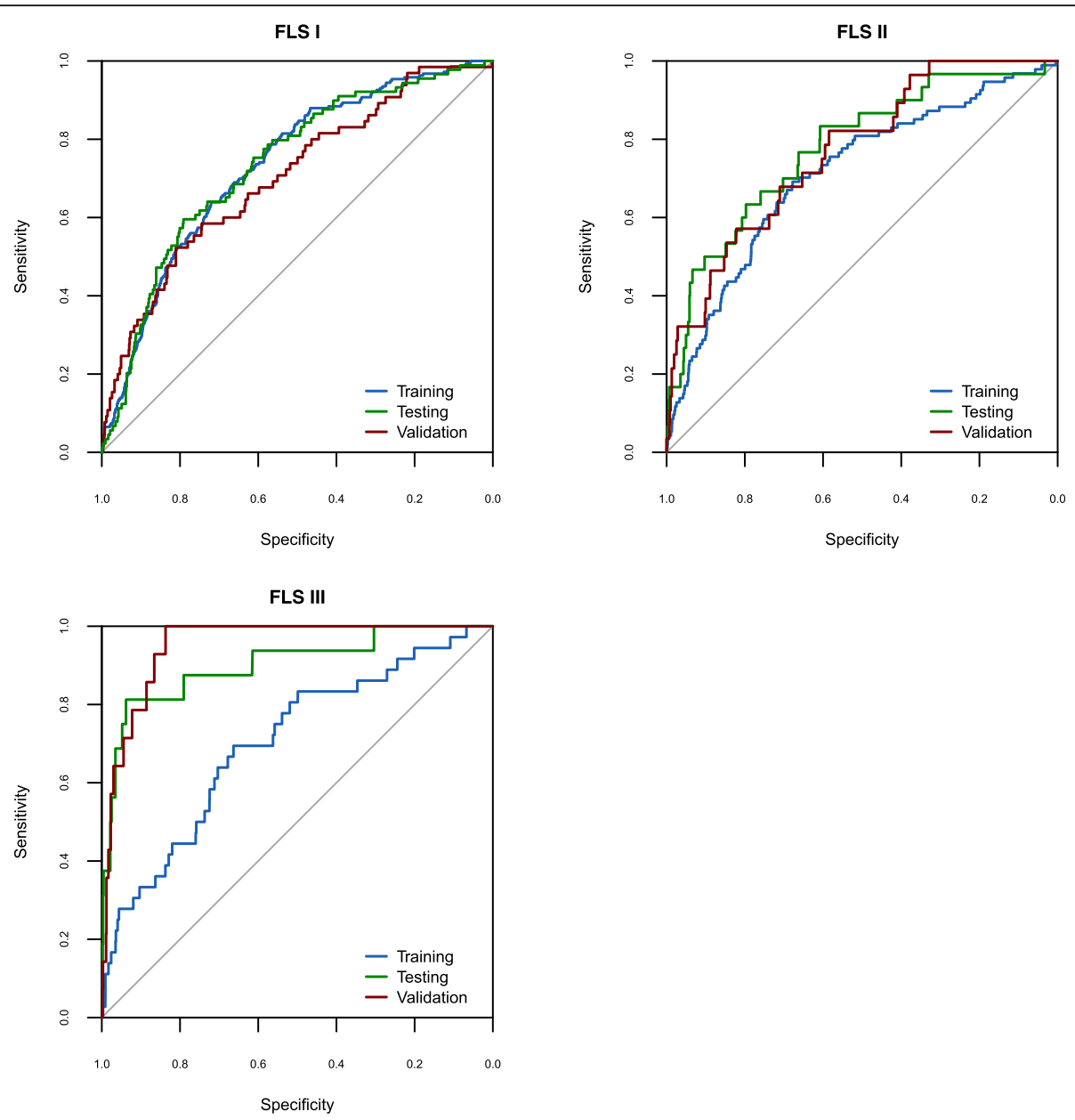

Fig. 1 FLS I, FLS II, FLS III Receiver operating characteristic (ROC) curves for training, testing and validation

likelihood of developing AKI (in stages 1, 2, or 3) and does not offer discriminated risk assessment, especially for more dangerous stages of AKI later during their hospital spell, including Stage 2 AKI (22-33\% mortality) and Stage 3 AKI (32-36\% mortality). This discriminated capability is provided by our risk assessment model

Table 5 AUC values with corresponding 95\% confidence intervals for all the models

\begin{tabular}{|c|c|c|c|c|c|c|}
\hline \multirow[t]{3}{*}{ Model } & \multicolumn{6}{|c|}{ Model Performance } \\
\hline & \multicolumn{2}{|c|}{ Training $^{a}$} & \multicolumn{2}{|c|}{ Testing $^{a}$} & \multicolumn{2}{|c|}{ Validation $^{\mathrm{a}}$} \\
\hline & $\mathrm{AUC}$ & $95 \% \mathrm{Cl}$ & AUC & $95 \% \mathrm{Cl}$ & AUC & $95 \% \mathrm{Cl}$ \\
\hline FLS I & 0.74 & $0.71-0.77$ & 0.73 & $0.68-0.79$ & 0.70 & $0.64-0.77$ \\
\hline MLR I & 0.73 & $0.70-0.77$ & 0.73 & $0.68-0.78$ & 0.70 & $0.64-0.77$ \\
\hline FLS II & 0.75 & $0.70-0.79$ & 0.78 & $0.69-0.87$ & 0.77 & $0.69-0.85$ \\
\hline MLR ॥ & 0.71 & $0.66-0.77$ & 0.78 & $0.69-0.87$ & 0.74 & $0.65-0.83$ \\
\hline FLS III & 0.79 & $0.71-0.86$ & 0.90 & $0.81-0.99$ & 0.95 & $0.92-0.98$ \\
\hline MLR III & 0.70 & $0.61-0.79$ & 0.82 & $0.68-0.96$ & 0.95 & $0.92-0.98$ \\
\hline
\end{tabular}

through the use of a fresh set of risk factors for each stage of AKI.

Each patient carries a risk of developing AKI at any time during hospitalisation. Research presented here quantifies that risk using information obtained at the point of admission and within the patient's first two days in hospital. Additionally, the capability to assess the risk of acquiring the more severe strands of acute kidney

Table 6 Sensitivity and specificity for all the models

\begin{tabular}{|c|c|c|c|c|c|c|c|c|c|}
\hline \multirow[t]{2}{*}{ Model } & \multirow[t]{2}{*}{ Cut-off } & \multicolumn{4}{|c|}{ Testing } & \multicolumn{4}{|c|}{ Validation } \\
\hline & & SN & $S P$ & PPV & NPV & SN & $S P$ & PPV & PV \\
\hline SI & (10) & 0.63 & 0.64 & 0.17 & 0.95 & 0.62 & 0.64 & 0.1 & 0.96 \\
\hline LR I & $4.2 \times 10^{-2}$ & 0.67 & 0.69 & 0.18 & 0.99 & 0.57 & 0.69 & 0.11 & 0.00 \\
\hline$S \|$ & $1.905 x$ & 0.7 & 0.7 & 0.07 & 0.99 & 0.61 & 0.73 & 0.06 & 0.9 \\
\hline 级 & $1.95 \times 10^{-2}$ & 0.7 & 0.7 & 0.07 & 0.99 & 0.61 & 0.72 & 0.06 & 0.93 \\
\hline SI & $8.019 \times 10$ & 0.81 & 0.81 & 0.07 & 1.0 & 1.0 & 0.83 & 0.08 & 1.0 \\
\hline LR III & $7.376 \times 10^{-3}$ & 0.75 & 0.75 & 0.05 & 0.99 & 1.0 & 0.79 & 0.06 & 1.0 \\
\hline
\end{tabular}



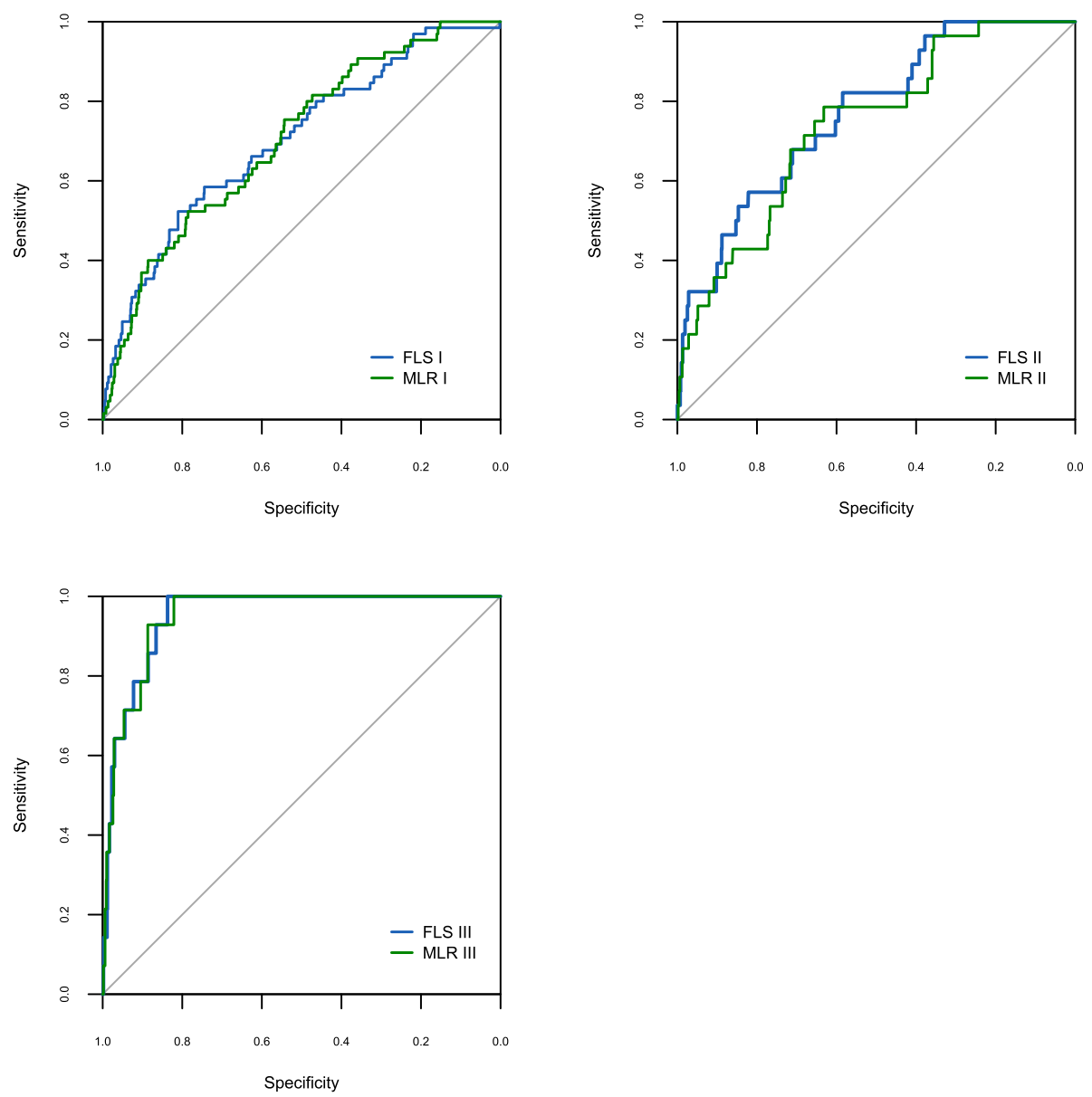

Fig. 2 FLS and MLR validation Receiver operating characteristic (ROC) curves

injury has never previously been attempted using a TSK FLS.

According to this research, use of FLS III could quickly have identified, within the first two days of a hospital spell, all 14 patients who went on to develop Stage 3 AKI during their stay. Moreover, with a specificity of $83 \%$, use of the same model would have falsely identified as "high risk" $17 \%$ of patients carrying low risk.

Susceptibility to either Stage 2 or Stage 3 is assessed by FLS II which correctly classifies $61 \%$ of high-risk patients and $73 \%$ of patients at low risk. Model performance for forecasting the development of any Stage of AKI, using FLS I, was marginally worse, predicting 62\% of cases that develop in RCHT and $64 \%$ of people not developing the disease.

The purpose behind development of FLS I was to forge comparison with risk tools previously assembled and, thereby assess the suitability of the selected input parameter set in predicting the patients at risk of developing AKI. Therefore, using the same model development technique but with either a refined or an expanded input parameter set, including prospectively collected data, enhanced predictive accuracy might be achieved.

FLS II and FLS III model in particular cannot be properly assessed against prior studies as this is the first attempt at quantifying the risk of developing specific Stages of AKI for a broad cohort of both medical and surgical inpatients. So, we believe our study has generated unrivalled accuracy in identifying patients at risk of Stage 3 AKI (AUC $=0.95,95 \%$ CI: 0.92-0.92). For Stage 2 or 3 AKI, FLS II model performance dropped comparatively (AUC $=0.77,95 \% \mathrm{CI}: 0.69-0.85$ ), to a level lower than the AUC $=0.9$ (95\% CI: 0.9-0.9) and AUC = 0.87 (95\% CI: 0.87-0.87) resulting from the application of a Gradient Boosting Machine algorithm forecasting patients developing Stage 2 AKI within 24 and $48 \mathrm{~h}$ respectively [22]. However, the approach presented here identifies patients at elevated risk of AKI up to a week ahead, allowing for a longer period of mitigating measures to be implemented. Likewise, a week is longer than the forecasting window employed in other research which used ward-monitored serum creatinine to predict onset of each Stage of AKI over the following 24 h [23]. 
Despite this shorter window, the AUC of 0.83 (95\% CI: 0.83-0.84) for Stage 3 AKI underperformed FLS III.

Another attempt at stratifying the risk of AKI development in-hospital used electronic patient data gathered $24 \mathrm{~h}$ either side of admission. Although categories employed to portray disease severity do not match AKI Stages used in this research and in clinical practice, an AUC of 0.75 (95\% CI: 0.73-0.76) resulted [24]. In the case of FLS I the accuracy of our approach fell slightly further (AUC $=0.70,95 \%$ CI: 0.64-0.77). However, the AUC for FLS I is comparable with a validation of an AKI Prediction score which was applied to identify development of hospital-acquired AKI in general medical and surgical admissions; for medical patients with no known baseline serum creatinine an AUC of up to 0.71 was achieved [25]. This contrasts to a number of studies which have looked to assess the risk of AKI in medical admissions. In analysing 898 patient records Roberts et al. generated AUC values of approximately 0.7 [10]. Marginally better levels of model performance were obtained by a study in Sussex, UK (AUC =0.72) [26]. Similarly, a study from a hospital in Kent, UK processed data from non-maternity, emergency admissions only and predicted the onset of AKI on admission (AUC $=0.75)$ and $72 \mathrm{~h}$ post admission $(\mathrm{AUC}=0.68)$ [27].

In addition to forecasting AKI up to a week prior to development, our study utilises data obtained 24 h either side of admission, thereby reducing computational burden. This contrasts to an approach which uses data gathered across varying windows, up to 30 days prior to admission [28]. Although the AUC reached 0.765, 0.73 and 0.7 (to predict AKI at times 1, 2 and 3 days prior respectively), patients in this cohort were limited to between 18 and 64 years old, and the researchers did not explore the applicability of the models for the elderly.

Our study performs less well than that employing up to five modelling techniques using data collected within the first $24 \mathrm{~h}$ of a hospital stay from patients aged 60 or over (AUC $=0.74,95 \%$ CI: 0.73-0.76) [29]. However, in addition to the age restriction, the research used variables which were not available electronically for all patient admissions at RCHT, e.g. BMI and Family History.

FLS I, however, has comparable performance to all of these studies, and uses data from both medical and surgical patients and both emergency and non-emergency.

According to our study data, mortality from AKI across our study period stood at 38\% for Stage 3 and 17\% for both AKI Stage 1 and AKI Stage 2. Given approximately $30 \%$ of cases are considered preventable, we propose that use of an accurate risk assessment tool for the development of in-hospital AKI Stage 3 could save lives. Based on the results presented in this study, the use of FLS III could evolve the potential to avoid some of these deaths.
The ability of FLS III to predict patients at low risk of AKI Stage 3 is not as good as it is for those at high risk. Falsely mitigating against some conditions, e.g. Deep Vein Thrombosis, can bring about unacceptable risks, i.e. bleeds. However, we consider the potential harm to the patient from inappropriate mitigation is likely to be sufficiently outweighed by the marginal risk caused by withholding measures. So, the use of a tool such as FLS III is worthy of health economic testing for the prevention of AKI Stage 3 in hospital.

A model of mitigation would include informing the patient's doctor, pharmacist, and nurse of their elevated risk. This would then bring forward a reassessment of the patient that includes:

- Reviewing their current condition and current diagnosis;

- considering alternative causes of illness (particularly searching for signs of sepsis);

- reviewing dates of recent and/or planned iodinated contrast scans;

- reviewing their current medication (particularly medication with an effect on blood pressure and renal haemo-dynamics);

- examining their fluid status and assessing the need for IV fluids [30]; and

- planning future blood tests and physiological observation monitoring.

These measures might be considered low-cost and add minimal risk for patients to whom they are unnecessarily introduced, including those falsely identified as being at high risk. The specificity results of this study indicate that mitigating measures will be unnecessarily introduced to roughly a third of patients carrying low risk who are expected to stay more than two days in hospital. In addition, given the lack of intrusiveness as these measures are introduced to patients, this is likely to be considered acceptable.

The performance of each FLS was assessed against MLR, a methodology more traditionally employed in developing risk assessment frameworks in healthcare. In all three models, the performance of FLS was at least as good as MLR, perhaps signalling that FLS could be employed more widely in health risk assessment and epidemiological research.

It is hypothesised that by identifying a patient's risk of developing AKI Stage 3 at the earliest point possible during a patient spell this will increase survival. Based on the data period within this study, the fraction of patients dying in hospital following a diagnosis of Stage 3 AKI during hospitalisation stands at $38 \%$ (25 out of 66 ). Assuming one fifth of these cases were preventable and FLS III would have early identified $80 \%$, use of this risk 
assessment tool could have prevented approximately 4 deaths during the same hospital stay.

Of 304 patients who went on to develop Stage 1 or Stage 2 AKI, 53 died. Assuming one fifth preventable and a FLS sensitivity of $60 \%$, a further 6 lives might have been saved with early risk assessment.

However, these claims should be tempered because later development of AKI can be a signature of a general end-of-life decline and greater mortality risk [24]. Nevertheless, due to the high levels of predictive accuracy achieved by research and given that $20 \%$ of cases of AKI developed in hospital are avoidable, it offers considerable potential to cut the in-hospital burden of the disease if accompanied by appropriate mitigating measures.

\section{Conclusions}

Acute Kidney Injury can develop in patients in hospital with the severest type, Stage 3, having a mortality rate of between 30 and $40 \%$. With appropriate mitigation, some cases can be avoided. We have used anonymised data taken from patients as they were admitted to hospital to create risk assessment models using Fuzzy Logic and multivariable logistic regression. The models were trained to recognise patterns in data and distinguish between those patients who went onto develop AKI any Stage, AKI Stage 2 and 3, or AKI Stage 3 and those who did not develop AKI at all. This was the first attempt at building separate models for the three currently recognised degrees of AKI severity and upon testing the models, our ability to predict AKI Stage 3 was very strong, albeit on a small number of cases. Subsequently, our view is given that AKI is preventable in some cases and any technology containing these models is designed to be deployed early on in a patient's stay, it has the potential to save lives.

\section{Additional file}

Additional file 1: The additional file contains: 1) Flow diagram of the fuzzy modelling algorithm, 2) additional information regarding patient characteristics, 3) FLS membership function parameters, rules and consequent parameters, 4) MLR models'. (DOCX 74 kb)

\section{Abbreviations}

ACEl: Angiotensin converting enzyme inhibitors; AKI: Acute Kidney Injury; AUC: Area under the curve; CKD: Chronic kidney disease; FL: Fuzzy logic; FLS: Fuzzy logic system; Hb: Haemoglobin; HCT: Haematocrit; KDIGO: Kidney Disease Improving Global Outcomes; MCV: Mean corpuscular volume; MLR: Multivariable logistic regression; MPV: Mean platelet volume; NCEPOD: National Confidential Enquiry into Patient Outcome and Death; NPV: Negative predictive value; NSAID: Non-steroidal anti-inflammatory drugs; PPV: Positive predictive value; RBC: Red blood count; RCHT: Royal Cornwall Hospitals NHS Trust; ROC: Receiver operating characteristic; SD: Standard deviation; SN: Sensitivity; SP: Specificity; TSK: Takagi-Sugeno-Kang;

VD: Vascular disease; WBC: White blood count
Funding

The Duchy Health Charity has funded this research.

\section{Availability of data and materials}

The data that support the findings of this study are available from RCHT but restrictions apply to the availability of these data, which were used after obtaining Research \& Development (R\&D) approval for the current study, and so are not publicly available. Data however can be made available with permission of RCHT

\section{Authors' contributions}

(AA) assisted in identifying electronically available risk factors. AA developed the risk models to assess patient risk of hospital acquired Acute Kidney Injury (AKI). AA took the lead in writing the Methods and Results Sections and Additional file 1 of the submitted manuscript, and assisted with editing the remaining sections of the manuscript. (SBT) helped compose the application for funding and recruited AA. SBT oversaw development of the mathematical models and assisted with interpretation of model outputs and helped edit the submitted manuscript. (PMU) identified the need to develop a hospital acquired AKI risk prediction tool based on electronically collected patient data available on admission. PMU helped compose the application for funding, editing submissions and presenting to the Funding committee. PMU applied clinical knowledge in identifying which risk factors were relevant to the research objectives. PMU helped with clinical interpretation of model output and edit the submitted manuscript. (SD), as a Renal Consultant, provided expert context in interpretation of the model output. SD took the lead in writing the Background section of the manuscript and, through specialist knowledge of research in Renal Medicine, helped tailor the Discussion to convey the significance of the research undertaken. (ASP) lead composition of the funding application and presented with PMU to the Funding committee. Following advice from PMU, ASP coordinated collation of the input and output data, reformatting and forwarding to AA. ASP drafted the remaining sections of the manuscript and this submission as well as assisting with editing. All authors read and approved the final manuscript.

\section{Ethics approval and consent to participate}

Not applicable. This study was given Trust approval in September 2015. This is before HRA approval was required (which came into operation) in April 2016. Sponsor approval was given in October 2015 even though the trust and sponsor were the same.

At the time, the process of obtaining NHS permissions, which was referred to as Research \& Development (R\&D) approval, relied upon the research department, within the individual trusts, undertaking 'site specific assessments'. These checks included inter alia ensuring the relevant approvals were in place (such as a favourable ethical opinion if applicable). After obtaining site approval from the R\&D offices the study would require sponsor approval (green light).

This study was classified as 'A study limited to working with data (specific project only)' (IRAS 191394). As the research data was anonymised it did not require Ethics approval.

\section{Consent for publication}

Not applicable.

\section{Competing interests}

The authors declare that they have no competing interests.

\section{Publisher's Note}

Springer Nature remains neutral with regard to jurisdictional claims in published maps and institutional affiliations.

\section{Author details}

${ }^{1}$ Centre for Implementation Science, Faculty of Health Sciences, University of Southampton, Southampton SO17 1BJ, UK. ${ }^{2}$ College of Engineering, Mathematics, and Physical Sciences, University of Exeter, Penryn, Cornwall, TR10 9FE, UK. ${ }^{3}$ Research, Development, and Innovation, Royal Cornwall Hospitals NHS Trust, Truro TR1 3HD, UK. 
Received: 9 June 2018 Accepted: 29 January 2019

Published online: 14 February 2019

\section{References}

1. Diesase K. Improving global outcomes (KDIGO) acute kidney injury work group: KDIGO clinical practice guideline for acute kidney injury. Kidney Int Suppl. 2012;2(1):1-138.

2. Acute Kidney Injury (AKI) Algorithm [https://www.england.nhs.uk/ akiprogramme/aki-algorithm/].

3. Wang HE, Muntner P, Chertow GM, Warnock DG. Acute kidney injury and mortality in hospitalized patients. Am J Nephrol. 2012;35(4):349-55.

4. Selby NM, Crowley L, Fluck RJ, McIntyre CW, Monaghan J, Lawson N, Kolhe NV. Use of electronic results reporting to diagnose and monitor AKI in hospitalized patients. Clin J Am Soc Nephrol. 2012;7(4):533-40.

5. Wallace K, Mallard AS, Stratton JD, Johnston PA, Dickinson S, Parry RG. Use of an electronic alert to identify patients with acute kidney injury. Clinical medicine. 2014;14(1):22-6.

6. Kerr M, Bedford M, Matthews B, O'Donoghue D. The economic impact of acute kidney injury in England. Nephrol Dial Transplant. 2014;29(7):1362-8.

7. Stewart J, Findlay G, Smith N, Kelly K, Mason M. Acute kidney injury: adding insult to injury. National Confidential Enquiry into Patient Outcome and Death. 2009.

8. Recommended Minimum Requirements of a Care Bundle for Patients with AKI in Hospital [https://www.thinkkidneys.nhs.uk/aki/wp-content/uploads/ sites/2/2015/12/AKI-care-bundle-requirements-FINAL-12.07.16.pdf].

9. Communities at Risk of Developing Acute Kidney Injury [https://www. thinkkidneys.nhs.uk/wp-content/uploads/2015/07/Communities-at-risk-ofdeveloping-AKI-Think-Kidneys-010715.pdf].

10. Roberts G, Phillips D, McCarthy R, Bolusani H, Mizen P, Hassan M, Hooper R, Saddler K, Hu M, Lodhi S. Acute kidney injury risk assessment at the hospital front door: what is the best measure of risk? Clin Kidney J. 2015;8(6):673-80.

11. Takagi T, Sugeno M. Fuzzy identification of systems and its applications to modeling and control. Systems, Man and Cybernetics, IEEE Transactions on. 1985;SMC-15(1):116-32.

12. Sugeno M, Kang GT. Structure identification of fuzzy model. Fuzzy Sets Syst. 1988;28:15-33.

13. Argyropoulos A. Soft sensor development and process control of anaerobic digestion. PhD Thesis. In: University of Exeter; 2013.

14. Metz CE: Basic principles of ROC analysis. In: Semin Nucl Med: 1978: Elsevier; 1978: 283-298.

15. Hosmer DW Jr, Lemeshow S, Sturdivant RX. Applied logistic regression, vol. 398: John Wiley \& Sons; 2013.

16. López-Ratón M, Rodríguez-Álvarez MX, Cadarso-Suárez C, Gude-Sampedro F. OptimalCutpoints: an R package for selecting optimal cutpoints in diagnostic tests. J Stat Softw. 2014;61(8):1-36.

17. Xavier R, Turck N, Hainard A, Tiberti N, Lisacek F, Sanchez J-C, Muller M. pROC: an open-source package for $\mathrm{R}$ and $\mathrm{S}+$ to analyse and compare ROC curves. BMC bioinformatics. 2011;12:77.

18. Sun R, Sosnoff JJ. Novel sensing technology in fall risk assessment in older adults: a systematic review. BMC Geriatr. 2018;18(1):14.

19. Georg H, Daniela D. Five myths about variable selection. Transpl Int. 2017; 30(1):6-10.

20. Saccenti E, Hoefsloot HCJ, Smilde AK, Westerhuis JA, Hendriks MMWB. Reflections on univariate and multivariate analysis of metabolomics data. Metabolomics. 2014;10(3):361-74.

21. Malhotra R, Siew ED. Biomarkers for the early detection and prognosis of acute kidney injury. Clin J Am Soc Nephrol. 2017;12(1):149-73.

22. Koyner $J L$, Carey KA, Edelson DP, Churpek MM. The development of a machine learning inpatient acute kidney injury prediction model* ${ }^{*}$ Crit Care Med. 2018;46(7):1070-7.

23. Koyner $\mathrm{L}$, Adhikari R, Edelson DP, Churpek MM. Development of a multicenter Ward-based AKI prediction model. Clin J Am Soc Nephrol. 2016;11(11):1935-43.

24. Matheny ME, Miller RA, Ikizler TA, Waitman LR, Denny JC, Schildcrout JS, Dittus RS, Peterson JF. Development of inpatient risk stratification models of acute kidney injury for use in electronic health records. Medical decision making : an international journal of the Society for Medical Decision Making. 2010;30(6):639-50.

25. Hodgson LE, Sarnowski A, Roderick PJ, Dimitrov BD, Venn RM, Forni LG. Systematic review of prognostic prediction models for acute kidney injury (AKI) in general hospital populations. BMJ Open. 2017;7(9):e016591.
26. Forni LG, Dawes T, Sinclair H, Cheek E, Bewick V, Dennis M, Venn R. Identifying the patient at risk of acute kidney injury: a predictive scoring system for the development of acute kidney injury in acute medical patients. Nephron Clinical Practice. 2013:123(3-4):143-50.

27. Bedford M, Stevens P, Coulton S, Billings J, Farr M, Wheeler T, Kalli M, Mottishaw T, Farmer C. Development of risk models for the prediction of new or worsening acute kidney injury on or during hospital admission: a cohort and nested study; 2016.

28. Cheng P, Waitman LR, Hu Y, Liu M. Predicting inpatient acute kidney injury over different time horizons: how early and accurate? AMIA Annual Symposium proceedings AMIA Symposium. 2017;2018:565-74.

29. Kate RJ, Perez RM, Mazumdar D, Pasupathy KS, Nilakantan V. Prediction and detection models for acute kidney injury in hospitalized older adults. BMC Medical Informatics and Decision Making. 2016;16(1):39.

30. Padhi S, Bullock I, Li L, Stroud M. Intravenous fluid therapy for adults in hospital: summary of NICE guidance. BMJ. 2013;347.
Ready to submit your research? Choose BMC and benefit from:

- fast, convenient online submission

- thorough peer review by experienced researchers in your field

- rapid publication on acceptance

- support for research data, including large and complex data types

- gold Open Access which fosters wider collaboration and increased citations

- maximum visibility for your research: over $100 \mathrm{M}$ website views per year

At BMC, research is always in progress.

Learn more biomedcentral.com/submissions 